\title{
Development of Non-Linear Guidance Algorithms for Asteroids Close-Proximity Operations
}

\author{
Roberto Furfaro ${ }^{1}$, Brian Gaudet ${ }^{2}$, Daniel R. Wibben ${ }^{3}$ \\ The University of Arizona, Tucson, AZ, USA \\ and \\ Jules $\mathrm{Simo}^{4}$ \\ The University of Strathclyde, Glasgow, UK
}

\begin{abstract}
In this paper, we discuss non-linear methodologies that can be employed to devise realtime algorithms suitable for guidance and control of spacecrafts during asteroid closeproximity operations. Combination of optimal and sliding control theory provide the theoretical framework for the development of guidance laws that generates thrust commands as function of the estimated spacecraft state. Using a Lyapunov second theorem one can design non-linear guidance laws that are proven to be globally stable against unknown perturbations with known upper bound. Such algorithms can be employed for autonomous targeting of points of the asteroid surface (soft landing, Touch-And-Go (TAG) maneuvers). Here, we theoretically derived and tested the Optimal Sliding Guidance (OSG) for close-proximity operations. The guidance algorithm has its root in the generalized ZEM/ZEV feedback guidance and its mathematical equations are naturally derived by a proper definition of a sliding surface as function of Zero-Effort-Miss and Zero-EffortVelocity. Thus, the sliding surface allows a natural augmentation of the energy-optimal guidance via a sliding mode that ensures global stability for the proposed algorithm. A set of Monte Carlo simulations in realistic environment are executed to assess the guidance performance in typical operational scenarios found during asteroids close-proximity operations. OSG is shown to satisfy stringent requirements for asteroid pinpoint landing and sampling accuracy.
\end{abstract}

\section{Introduction}

$\mathrm{O}$ ver the past few years, there has been a strong interest in sending robotic spacecrafts to small bodies orbiting around the Sun. Such bodies include comets and Near Earth Asteroids (NEAs). Over the past few billions years, such objects have been minimally processed and detailed remote mapping and in-situ sampling may provide scientists with opportunities to unveil the early history of the solar system ${ }^{1}$. Aside the extremely valuable contribution that NEAs missions would provide to the global understanding of the origin of the Solar System, such robotic missions would help characterizing and quantifying the amount of extraterrestrial natural resources ${ }^{2}$ as well as quantifying the risk that such objects may collide with planet Earth ${ }^{3}$. In May 2011, NASA announced the selection of OSIRIS REx asteroid sample return mission ${ }^{4}$ as part of the New Frontier 3 program. Launching in 2016, the spacecraft is expected to arrive at the 1999 RQ36 "Bennu" asteroid in late 2018. After performing close proximity mapping operations for approximately 18 months, the spacecraft will descend toward the surface to capture the sample with the goal of returning it safely to Earth by mid-2023.

\footnotetext{
${ }^{1}$ Assistant Professor, Department of Systems and Industrial Engineering, Department of Aerospace and Mechanical Engineering, 1127 E. James E. Rogers Way, Tucson, AZ 85721

${ }^{2}$ Research Engineer, Department of Systems and Industrial Engineering, 1127 E. James E. Rogers Way, Tucson, AZ 85721

${ }^{3} \mathrm{Ph}$. D. Student, Department of Systems and Industrial Engineering, 1127 E. James E. Rogers Way, Tucson, AZ 85721

${ }^{4}$ Academic Visitor, Department of Mechanical and Aerospace Engineering, University of Strathclyde, Glasgow, UK
} 
Generally, close-proximity operations around small celestial objects are extremely challenging. Indeed, the dynamics of the spacecraft is complicated by a number of factors including 1) irregular shape and mass distribution of the object; 2) weak and uncertain gravitational field; and 3) perturbations due to solar radiation pressure. As a result, spacecraft trajectories around such bodies are generally complex and non-periodic. Moreover, the stability of the motion is guaranteed for a limited set of latitudes 5 . Furthermore, close orbit operations are characterized by general communication time delays, a rapid rotational dynamics (order of hours), and the unknown and changing surface properties from illumination variation and surface conditions. In such challenging environment, sustained investigations of small bodies require that the spacecraft seamlessly transitions from one state to another to gain different vantage observational points. For example, during the course of a small body mission, the spacecraft may be required to hover around various points around the asteroid and land repeatedly on different surface locations to completely characterize the nature of the small body under investigation. Although current practice involving closeproximity operations around asteroids require heavy human intervention, autonomous operations, including guidance and orbit control, may be at least desirable.

To successful execute autonomous close-proximity operations around asteroids, the spacecraft must be equipped with a properly designed Guidance, Navigation and Control (GNC) subsystem. The latter must be able to a) autonomously process information coming from sensors (e.g. optical cameras, LIDAR) to estimate in real-time position and velocity relative to the small body, b) process the current spacecraft state to generate an real-time acceleration command that drives the spacecraft toward the desired position and c) allocate the commanded acceleration signal to the proper thrusters to implement the desired maneuver and achieve the desired target state. Integrated navigation and control systems have been recently proposed and studied. Misuet al. ${ }^{6}$ proposed an autonomous rendezvous guidance scheme based on feature extraction and inheritance. The GNC methodology is based on fixation-point inheritance, where the spacecraft descend toward the asteroid targeted point by tracking and autonomously renewing fixation-points. The proposed system uses optical images coupled with an extended Kalman Filter to estimate the state and an ad-hoc, on-off thruster control logic to drive the spacecraft to the target. Shuanget al. ${ }^{7}$ proposed an integrated scheme for close-proximity operations: first an autonomous navigation algorithm based on feature tracking technology is devised. Then, two guidance control schemes (i.e. error phase analysis method and Proportional-Derivative (PD) plus Pulse-Width Pulsed Frequency (PWPF)) were studied and simulated to verify performances. More recently,Bhaskaranet al. ${ }^{8}$ coupled two independent frameworks that formed the basis of an autonomous navigation system for landing on small bodies. The first consisted in a general autonomous navigation framework that incorporates trajectory propagation, observables and partials generation as well as maneuver design and targeting. The second aspect dealt with shape modeling and landmark tracking scheme which provides vectors from the spacecraft to the surface to be used as data by the OBIRON (On-Board Image Registration for Optical Navigation) navigation process.

Most of the above mentioned papers focused mainly on the navigation aspects of the problem. Indeed, standard trajectory reference tracking algorithms have been implemented to verify the ability of the GNC system to drive the spacecraft toward the desired state. Nevertheless, advancements in non-linear control theory may be employed to generate flexible, yet robust guidance algorithms for close proximity operations. Furfaro et al. ${ }^{9}$ proposed a nonlinear guidance algorithm for asteroid landing. Based on Higher Order Sliding Control $\left(\mathrm{HOSC}^{10}\right)$, the algorithm is shown to perform well in an uncertain dynamical environment. In this paper, we show how to develop a class of guidance algorithms for real-time, close-proximity operations that are energy-optimal yet robust against unmodelled dynamics and uncertain parameters. We propose the developmentof theOptimal Sliding Guidance (OSG) for close proximity operations which can be employed to transition from two different states, including from and to the asteroid surface. OSG is based on the generalized ZEM/ZEV (Zero Effort Miss/Zero Effort Velocity) feedback guidance ${ }^{11}$. Rooted in optimal control theory and originally derived by Battin ${ }^{12}$ and $\mathrm{D}^{\text {'souza }}{ }^{13}$ for planetary landing, the ZEM/ZEV guidance is demonstrated to be energy-optimal under constant and time-dependent gravitational field ${ }^{11}$. Hawkins et al. ${ }^{14}$ adapted the ZEM/ZEV guidance algorithm to asteroid close-proximity operations showing how to theoretical derive the guidance equations. Here, we couple optimal and sliding control theory to show how to robustify the ZEM/ZEV feedback guidance by augmenting it via a properly defined sliding control mode. The latter yields a guidance algorithm that is globally stable in an uncertain dynamical environment for which an upper bound of the perturbing acceleration is known. OSG is naturally derived by defining the sliding surface as function of ZEM and ZEV. Lyapunov theory can be effectively employed to demonstrate the guidance global stability. OSG is tested in a realistic simulation environment to demonstrate the performance of the algorithm for close proximity operations around RQ36 "Bennu" which is the target of the NASA OSIRIS REx Asteroid Sample Return Mission. 


\section{Methodology}

\section{A. Close-Proximity Spacecraft Dynamical Model}

In formulating the spacecraft guidance problem for close-proximity operations around asteroids, we model the spacecraft dynamics near the asteroid using a two-body gravitational model (spacecraft has negligible mass). The equations of motion for the spacecraft in a uniformly rotating, asteroid-fixed Cartesian coordinate frame having the origin at the asteroid center of mass are written as follows:

$$
\begin{gathered}
\dot{\boldsymbol{r}}_{L}=\boldsymbol{v}_{L} \\
\boldsymbol{v}_{L}=2 \boldsymbol{\omega} \times \boldsymbol{v}_{L}+\boldsymbol{\omega} \times \boldsymbol{\omega} \times \boldsymbol{r}_{L}+\boldsymbol{g}\left(\boldsymbol{r}_{L}\right)+\boldsymbol{a}_{\text {COMM }}+\boldsymbol{p}
\end{gathered}
$$

Here, $\boldsymbol{r}_{L}=[x, y, z]^{T}$ is the position vector in the body-fixed rotating frame, $\boldsymbol{v}_{L}=\left[v_{x}, v_{y}, v_{z}\right]^{T}$ is the velocity vector, $\boldsymbol{g}\left(\boldsymbol{r}_{L}\right)=\left[g_{x}, g_{y}, g_{z}\right]^{T}$ is the local gravitational field, $\boldsymbol{a}_{\text {COMM }}=\left[a_{c x}, a_{c y}, a_{c z}\right]^{T}$ is the acceleration command and $\boldsymbol{p}=\left[p_{x}, p_{y}, p_{z}\right]^{T}$ is the perturbing acceleration, accounting for unmodeled/unknown forces (e.g. gravity field inaccuracies, solar radiation pressure and nth-body perturbations).

For this analysis, it is assumed that the shape of the asteroid can modeled as a triaxial ellipsoid allowing analytical determination of the asteroid gravitational field. The gravitational field can be expressed as a partial derivative of the potential field, i.e. $\boldsymbol{g}\left(\boldsymbol{r}_{\boldsymbol{L}}\right)=\partial V / \partial \boldsymbol{r}_{\boldsymbol{L}}{ }^{T}$.

The equations of motion can be explicitly written in their scalar form:

$$
\begin{gathered}
\dot{x}=v_{x} \\
\dot{y}=v_{y} \\
\dot{z}=v_{z} \\
\dot{v}_{x}=2 \omega v_{y}+\omega^{2} x+\frac{\partial V}{\partial x}+a_{C x}+p_{x} \\
\dot{v}_{y}=-2 \omega v_{x}+\omega^{2} y+\frac{\partial V}{\partial y}+a_{C y}+p_{y} \\
\dot{v}_{z}=\frac{\partial V}{\partial z}+a_{C z}+p_{z}
\end{gathered}
$$

The mathematical model described in Eq. (1-8) is employed to derive the guidance equations. In the development of the guidance law, the mass of the spacecraft is assumed to be constant. However, in more realistic Monte Carlo simulations required to test the performance of the proposed OSG law, the model is upgraded to account for mass variation as given by the classical rocket equation:

$$
\dot{m}=-\frac{\|T\|}{I_{s p} g_{c}}
$$

Where $\|\boldsymbol{T}\|=\sqrt{T_{x}^{2}+T_{y}^{2}+T_{z}^{2}}$ is the magnitude of the overall thrust vector $\boldsymbol{T}=\left[T_{x}, T_{y}, T_{z}\right]^{T}$, and $g_{0}$ is the gravitational acceleration at sea level. The thrust vector is linked to the acceleration command according to the conventional thrust-to-mass ratio $\boldsymbol{a}_{\text {Сомм }}=\boldsymbol{T} / \mathrm{m}$ relationship. 


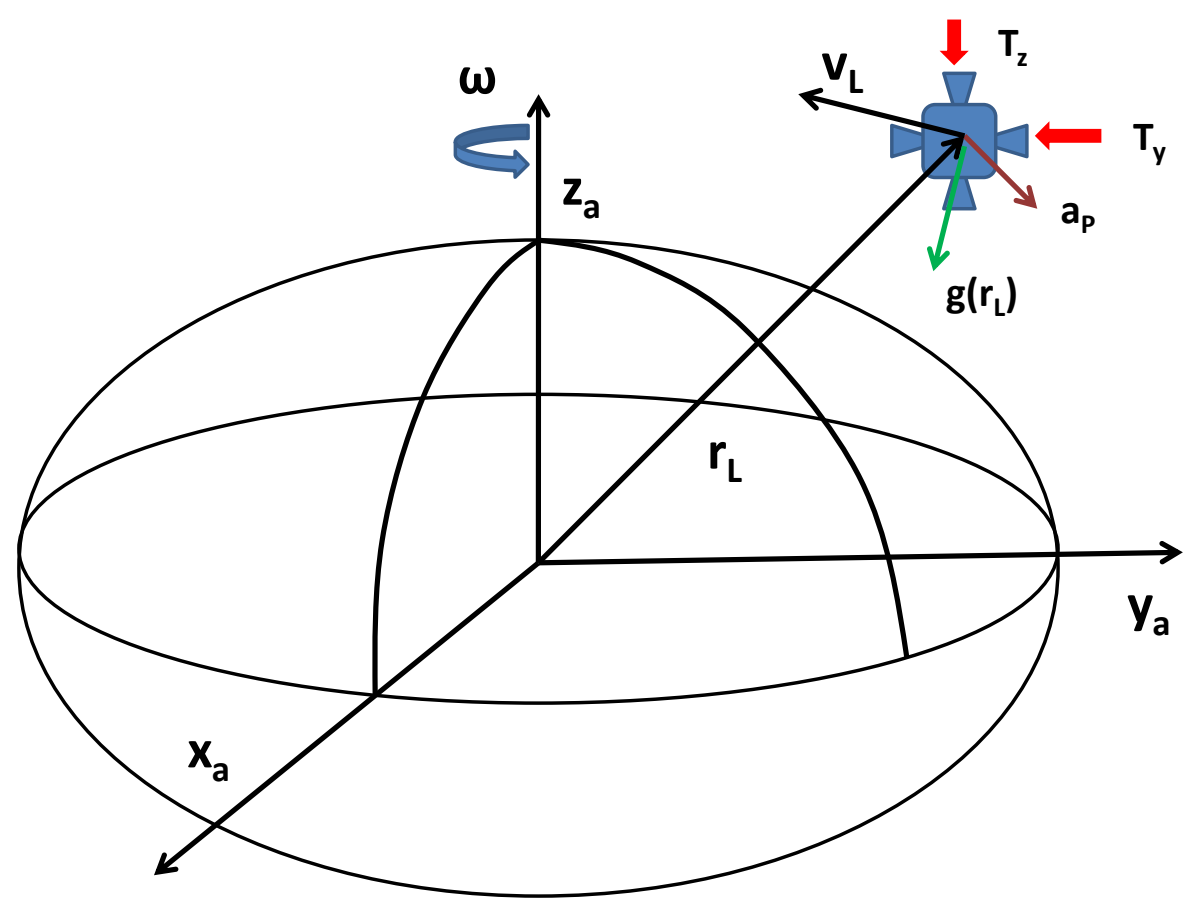

Figure 1.Free-body diagram representing the forces acting on the spacecraft in a body-fixed asteroid reference frame

\section{B.Non-linear Guidance Algorithms Development}

The goal of this paper is to present a set of targeting and real-time guidance algorithms for asteroid closeproximity operations. Such algorithms are designed by combining some known results from optimal control theory as applied to the landing problem ${ }^{10,12}$ and advancements in non-linear sliding control theory ${ }^{15}$. The sliding control approach to targeting requires a geometric understanding of the control problem. The idea of using sliding control modes for real-time guidance is rooted into employing "surfaces" to drive the dynamical system to the desired state. A sliding surface is defined as a linear combination of the state error and/or its derivative. Whenever the system state (position and velocity) is locked on the sliding surface, the dynamical system is tracking the desired reference signal with null error.

Proper development of sliding-based guidance algorithms require the definition of a suitable guidance model which is presented next.

1. Guidance model: Zero-Effort Miss (ZEM) and Zero-Effort Velocity (ZEV) errors

The physical model employed to develop the guidance algorithm is a 3-DOF model similar to the one presented in the previous section (see Eq. (1)-(8)). However, the guidance model does not account for a mass variation. The equations can be synthetically represented as follows:

$$
\begin{gathered}
\frac{d}{d t} \boldsymbol{r}_{L}=\boldsymbol{v}_{L} \\
\frac{d}{d t} \boldsymbol{v}_{l}=\boldsymbol{a}_{L}(t)=\boldsymbol{g}\left(\boldsymbol{r}_{L}, t\right)+\boldsymbol{a}_{\text {COMM }}(t)
\end{gathered}
$$

Here, the vector $\boldsymbol{g}\left(\boldsymbol{r}_{L}, t\right)=\mathbf{2} \boldsymbol{\omega} \times \boldsymbol{v}_{L}+\boldsymbol{\omega} \times \boldsymbol{\omega} \times \boldsymbol{r}_{L}+\partial V / \partial \boldsymbol{r}_{\boldsymbol{L}}{ }^{T}$ represents all forces acting on the spacecraft (except for the thrust) whereas асомм is the acceleration command (i.e. thrust-to-mass ratio) that drives the 
spacecraft to the desired state. Eq. (10)-(11) can be integrated starting from knowledge of position and velocity at time $t$ to formally determine position and velocity of the spacecraft at a specified final time $t_{f}$ :

$$
\begin{gathered}
\boldsymbol{v}_{L}\left(t_{f}\right)=\boldsymbol{v}_{L}(t)+\int_{t}^{t_{f}}\left(\boldsymbol{g}\left(\boldsymbol{r}_{L}, \tau\right)+\boldsymbol{a}_{\text {COMM }}(\tau)\right) d \tau \\
\boldsymbol{r}_{L}\left(t_{f}\right)=\boldsymbol{r}_{L}(t)+\boldsymbol{v}_{L}(t) t_{g o}+\int_{\mathrm{t}}^{t_{f}} \int_{\tau^{\prime}}^{t_{f}}\left(\boldsymbol{g}\left(\boldsymbol{r}_{L}, \tau^{\prime}\right)+\boldsymbol{a}_{\text {COMM }}\left(\tau^{\prime}\right)\right) d \tau^{\prime} d \tau
\end{gathered}
$$

Here $\mathrm{t}_{\mathrm{go}}=\mathrm{t}_{\mathrm{f}}-\mathrm{t}$ is the time-to-go, i.e. the time required to reach the desired position (target) with the desired velocity. Next, we define the following quantities:

Definition \#1: Given the time t, we define the Zero-Effort Miss (ZEM) as the distance (vector) the spacecraft will miss the target if no acceleration command (guidance) is generated after t:

$$
\boldsymbol{Z E M}(t)=\boldsymbol{r}_{L f}-\boldsymbol{r}_{L}\left(t_{f}\right), \quad \boldsymbol{a}_{\text {COMM }}(\tau)=\mathbf{0}, \tau \in\left[t, t_{f}\right]
$$

Definition \#2: Given the time t, we define the Zero-Effort Velocity (ZEV) as the error in velocity at the final time, if no acceleration command (guidance) is generated after t, i.e.

$$
\boldsymbol{Z E V}(t)=\boldsymbol{v}_{L f}-\boldsymbol{v}_{L}\left(t_{f}\right), \quad \boldsymbol{a}_{\text {СомM }}(\tau)=\mathbf{0}, \tau \in\left[t, t_{f}\right]
$$

Here, $\boldsymbol{r}_{L f}$ and $\boldsymbol{v}_{L f}$ are the desired position and velocity at the final time. Both $\boldsymbol{Z E \boldsymbol { M }}(t)$ and $\boldsymbol{Z E V}(t)$ can be explicitly expressed as functions of the current position, velocity and time-to-go by substituting Eq. $(12,13)$ with $\mathbf{a}_{\text {сомm }}=\mathbf{0}$ into Eq.(14) and Eq.(15):

$$
\begin{gathered}
\boldsymbol{Z} \boldsymbol{E} \boldsymbol{V}(t)=\boldsymbol{v}_{L f}-\boldsymbol{v}_{L}(t)-\int_{t}^{t_{f}} \boldsymbol{g}\left(\boldsymbol{r}_{L}, \tau\right) d \tau \\
\boldsymbol{Z E \boldsymbol { M }}(t)=\boldsymbol{r}_{L f}-\boldsymbol{r}_{L}(t)-\boldsymbol{v}_{L}(t) t_{g o}-\int_{\mathrm{t}}^{\mathrm{t}_{f}} \int_{\tau^{\prime}}^{t_{f}} \boldsymbol{g}\left(\boldsymbol{r}_{L}, \tau^{\prime}\right) d \tau^{\prime} d \tau
\end{gathered}
$$

\section{Optimal guidance for lunar landing: Basic equations}

The basis of our algorithm development is the ability to generate an optimal guidance law as a function of $\boldsymbol{Z} \boldsymbol{E M}$ and $\boldsymbol{Z} \boldsymbol{E V}$. Indeed, given the actual spacecraft position and velocity, both quantities can be estimated on-line by the numerical integration of the (unperturbed) equations of motion as functions of the time-to-go and the targeted conditions. One of the key ingredients is the ability to obtain a closed loop guidance law that minimizes the overall guidance effort, i.e. a guidance law thatminimizes the overall acceleration command. The problem can be formulated as follows:

Find the $\boldsymbol{a}_{\text {Сомм }}$ as a function of $\boldsymbol{Z} \boldsymbol{E} \boldsymbol{M}(t)$ and $\boldsymbol{Z} \boldsymbol{E} \boldsymbol{V}(t)$ that minimizes the following performance index:

$$
J\left(\boldsymbol{a}_{\text {Сом }}\right)=\int_{t}^{t_{f}} \boldsymbol{a}_{\text {Сом }}(\tau)^{T} \boldsymbol{a}_{\text {Сом }}(\tau) d \tau
$$

Subject to Eq. $(11,12)$ as physical constraints, with initial conditions (at time $t) r(t)$ and $v(t)$ and final conditions (at time $\left.t_{f}\right) r_{L}$ and $v_{L}$.

The acceleration command is assumed to be unconstrained, i.e. the thrust generated by the propulsion system is unbounded. The problem can be solved by either applying the Pontryagin Minimum Principle (PMP) to determine the necessary conditions for the existence of an optimal solution (Two-Point Boundary Value Problem, TPBVP) or by a direct application of calculus of variations (see appendix A). It is found that the acceleration command is linear in time, i.e.:

$$
\boldsymbol{a}_{\text {СOMM }}(t)=\boldsymbol{A}_{1} t-\boldsymbol{A}_{2}
$$




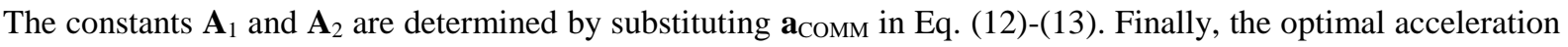
command can be expressed as a function of $\mathbf{Z E M}(\mathrm{t}), \mathbf{Z E V}(\mathrm{t})$ and $\mathrm{t}_{\mathrm{go}}$ as follows:

$$
\boldsymbol{a}_{\text {СомM }}(t)=\frac{k_{R}}{t_{g o}^{2}} \boldsymbol{Z E} \boldsymbol{M}(t)+\frac{k_{V}}{t_{g o}} \boldsymbol{Z E V}(t)
$$

Where $\mathrm{k}_{\mathrm{R}}=6$, and $\mathrm{k}_{\mathrm{V}}=-2$ are the optimal guidance gains (details of the derivation are presented in appendix A).

The methodology employed to determine the optimal guidance law as a function of $\boldsymbol{Z E} \boldsymbol{M}$ and $\boldsymbol{Z} \boldsymbol{E V}$ is very similar to the analysis presented by D'Souza ${ }^{13}$, who derived the optimal acceleration command for a power landing descent as a function of error in position (actual position minus target position), velocity (actual velocity minus target velocity) and time-to-go. Both formulations do not impose any constraints in term of maximum thrust or minimum altitude. Nevertheless, both algorithms are easy to implement and mechanize which may justify the attractiveness of the guidance approach.Numerical simulations of the closed-loop trajectories may be analyzed aposteriori to verify that both constraints are never violated or that the guidance algorithm works (i.e. guides the spacecraft to the target) even in the presence of thrust saturation.

\section{Sliding Control Theory}

The sliding control methodology is an elementary approach to robust control ${ }^{15}$. Intuitively, it is based on the observation that it is much easier to control non-linear and uncertain $1^{\text {st }}$ order systems (i.e. described by $1^{\text {st }}$ order differential equations) than $\mathrm{n}^{\text {th }}$-order systems (i.e. described by $\mathrm{n}^{\text {th }}$-order differential equations). Generally, if a transformation is found such that an $\mathrm{n}^{\text {th }}$-order problem can be replaced by a $1^{\text {st }}$ order problem, it can be shown that, for the transformed problem, perfect performance can be in principle achieved in presence of parameter inaccuracy. As a drawback, such performance is generally obtained at the price of higher control activity.

Consider the following single-input $\mathrm{n}^{\text {th }}$-order dynamical system:

$$
\frac{d^{n}}{d t^{n}} x=f(\boldsymbol{x})+b(\boldsymbol{x}) u
$$

Here, $x$ is the scalar output, $\mathrm{u}$ is the control variable and $\boldsymbol{x}=\left[x, \dot{x}, \ldots \ldots, x^{(n)}\right]^{T}$ is the state vector. Both $f(\boldsymbol{x})$, which describes the non-linear system dynamics, and the control gain $b(\boldsymbol{x})$ are not exactly known. Assuming that both $f(\boldsymbol{x})$ and $b(\boldsymbol{x})$ have a known upper bound, the sliding control goal is to get the state $\boldsymbol{x}$ to track the desired state $\boldsymbol{x}_{\boldsymbol{d}}=\left[x_{d}, \dot{x}_{d}, \ldots ., x_{d}^{(n)}\right]^{\boldsymbol{T}}$ in presence of model uncertainties. The time varying sliding surface is introduced as a function of the tracking error $\widetilde{\boldsymbol{x}}=\boldsymbol{x}-\boldsymbol{x}_{\boldsymbol{d}}$ by the following scalar equation:

$$
s(\boldsymbol{x}, t)=\left(\frac{d}{d t}+\lambda\right)^{n-1} \widetilde{\boldsymbol{x}}=0
$$

For example, if $\mathrm{n}=2$ we obtain:

$$
s(\boldsymbol{x}, t)=\dot{\tilde{\boldsymbol{x}}}+\lambda \widetilde{\boldsymbol{x}}=0
$$

Importantly, $\lambda$ is a strictly positive parameter. With the definitions in Eq. (22) and Eq. (23), the tracking problem is reduced to the problem of forcing the dynamical system in Eq. (21) to remain on the time-varying sliding surface. Clearly, tracking an n-dimensional vector $\boldsymbol{x}_{\boldsymbol{d}}$ has been reduced to the problem of keeping the scalar sliding surface to zero, i.e. the problem has been reduced to a $1^{\text {st }}$ order stabilization problem in $\mathrm{s}$. The simplified $1^{\text {st }}$ order stabilization problem can be now achieved by selecting a control law such that outside the sliding surface the following is satisfied:

$$
\frac{1}{2} \frac{d}{d t} s^{2} \leq-\eta|s|
$$

Here, $\eta$ is a strictly positive constant. Eq. (24), also called the "sliding condition", explicitly states that the distance from the sliding surface decreases along all system trajectories. Generally, constructing a control law that satisfies the sliding condition is fairly straightforward. For example, using the Lyapunov direct method one can select a candidate Lyapunov function as follows: 


$$
V(s)=\frac{1}{2} s^{T} S
$$

With $V(0)=0$ and $V(s)>0$ for $s>0$. By taking the derivative of Eq. (25), it is easily concluded that the sliding condition (Eq. (24)) is satisfied. The control law is generally obtained by substituting the sliding control definition, Eq. (23), and the system dynamical equations, Eq. (21), into Eq. (24).

\section{C.Optimal Sliding Guidance (OSG) Design}

The mathematical expression of the acceleration command is fairly simple and may be attractive for direct implementation on the on-board guidance computer. However, the optimal guidance, as derived, does not account for unmodeled disturbances which may negatively affect its performance. Here, the overall goal is to integrate a non-linear sliding control mode into the optimal guidance law to produce a robust, non-linear guidance algorithm.

To implement the sliding control approach into the optimal guidance framework and derive the Optimal Sliding Guidance (OSG) equations, we begin by defining a sliding surface (vector) as a function of ZEM and ZEV as follows:

$$
\boldsymbol{s}=\boldsymbol{Z E V}+\tilde{\lambda} \boldsymbol{Z E M}
$$

Clearly, the surface goes to the null value as ZEM and ZEV both approach zero. Subsequently, the idea is to

construct the guidance law in such a way that the system is always driven to the sliding surface. Therefore, we consider the dynamics of the sliding surface, i.e. take the derivative of Eq.(21) and substitute the expressions for the derivative of ZEM and ZEV:

$$
\frac{d}{d t} \boldsymbol{s}=\frac{d}{d t} \boldsymbol{Z E V}+\tilde{\lambda} \frac{d}{d t} \boldsymbol{Z E} \boldsymbol{M}=-\left(1+\tilde{\lambda} t_{g o}\right) \boldsymbol{a}_{\text {СОМM }}
$$

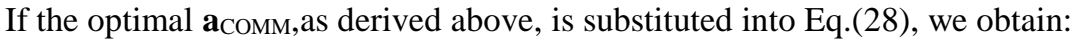

$$
\frac{d}{d t} \boldsymbol{s}=-\left[\left(1+\tilde{\lambda} t_{g o}\right) \frac{k_{V}}{t_{g o}} \boldsymbol{Z E V}+\left(1+\tilde{\lambda} t_{g o}\right) \frac{k_{R}}{t_{g o}^{2}} \boldsymbol{Z E} \boldsymbol{M}\right]=-K(t)(\boldsymbol{Z E V}+\tilde{\lambda} \boldsymbol{Z} \boldsymbol{E} \boldsymbol{M})=-K(t) \boldsymbol{s}
$$

The following relationships between the parameters can be easily found:

$$
\begin{gathered}
K(t)=\left(1+\tilde{\lambda} t_{g o}\right) \frac{k_{V}}{t_{g o}} \\
\tilde{\lambda} K(t)=\left(1+\tilde{\lambda} t_{g o}\right) \frac{k_{R}}{t_{g o}^{2}} \\
\tilde{\lambda}=\frac{k_{R}}{k_{V} t_{g o}}
\end{gathered}
$$

The sliding surface behaves as a non-linear first order system and its dynamics depend explicitly on the time-togo or $t_{F}-t$. Consequently, the system has the properties to reach the surface in a finite time which occurs exactly when $t_{F}=t$ or at the landing point. Thus the surface is reached for the first time at the landing point and chattering is avoided (see Appendix B for a detailed analysis of the sliding surface dynamics). The sliding mode is incorporated into the optimal guidance law to guarantee that the sliding surface behaves as follows:

$$
\frac{d}{d t} \boldsymbol{s}=-K(t) \boldsymbol{s}-\frac{\Phi}{t_{g o}} \operatorname{sign}(\boldsymbol{s})
$$

Here, $\Phi=$ const $>0$. The OSG equations are subsequently determined: 


$$
\boldsymbol{a}_{\text {СОMM }}(t)=\frac{k_{R}}{t_{g o}^{2}} \boldsymbol{Z} \boldsymbol{E M}(t)+\frac{k_{V}}{t_{g o}} \boldsymbol{Z} \boldsymbol{E} \boldsymbol{V}(t)-\frac{\Phi}{t_{g o}} \operatorname{sign}(\boldsymbol{s})
$$

The Lyapunov's second method is now employed to show that the OSG is globally stable and robust against perturbations. Consider the following quadratic function as a candidate Lyapunov function:

$$
V=\frac{1}{2} s^{T} s=\frac{1}{2}(\boldsymbol{Z E V}+\tilde{\lambda} \boldsymbol{Z E} \boldsymbol{M})^{T}(\boldsymbol{Z E V}+\tilde{\lambda} \boldsymbol{Z} \boldsymbol{E} \boldsymbol{M})
$$

Differentiating with respect to time, we obtain:

$$
\frac{d}{d t} V=\boldsymbol{s}^{T} \frac{d}{d t} \boldsymbol{S}=\boldsymbol{s}^{T}\left(\frac{d}{d t} \boldsymbol{Z} \boldsymbol{E} \boldsymbol{V}+\frac{k_{V}}{k_{R} t_{g o}} \frac{d}{d t} \boldsymbol{Z} \boldsymbol{E} \boldsymbol{M}\right)
$$

Inserting the expressions for the derivative of $\mathbf{Z E M}$ and $\mathbf{Z E V}$ :

$$
\begin{gathered}
\frac{d}{d t} V=\boldsymbol{s}^{T} \frac{d}{d t} \boldsymbol{s}=\boldsymbol{s}^{T}\left(-\boldsymbol{a}_{\text {COMM }}+\boldsymbol{p}(t)-\frac{k_{R}}{k_{V}}\left(\boldsymbol{a}_{\text {COMM }}-\boldsymbol{p}(t)\right)\right)= \\
=\boldsymbol{s}^{T}\left(-\left(\frac{k_{R}+k_{V}}{k_{V}}\right) \boldsymbol{a}_{\text {COM }}+\left(\frac{k_{R}+k_{V}}{k_{V}}\right) \boldsymbol{p}(t)\right)= \\
=\boldsymbol{s}^{T}\left(-\left(\frac{k_{R}+k_{V}}{k_{V}}\right)\left(\frac{k_{R}}{t_{g o}^{2}} \boldsymbol{Z} \boldsymbol{E} \boldsymbol{M}(t)+\frac{k_{V}}{t_{g o}} \boldsymbol{Z} \boldsymbol{E} \boldsymbol{V}(t)-\frac{\Phi}{t_{g o}} \operatorname{sign}(\boldsymbol{s})\right)+\left(\frac{k_{R}+k_{V}}{k_{V}}\right) \boldsymbol{p}(t)\right)= \\
=\boldsymbol{s}^{T}\left(-\left(\frac{k_{R}+k_{V}}{t_{g o}}\right)\left(\boldsymbol{Z} \boldsymbol{E} \boldsymbol{V}(t)+\frac{k_{R}}{k_{V} t_{g o}} \boldsymbol{Z} \boldsymbol{E} \boldsymbol{M}(t)\right)-\left(\frac{k_{R}+k_{V}}{k_{R}}\right)\left(-\frac{\Phi}{t_{g o}} \operatorname{sign}(\boldsymbol{s})+\boldsymbol{p}(t)\right)\right)= \\
=\left(\frac{k_{R}+k_{V}}{t_{g o}}\right) \boldsymbol{s}^{T} \boldsymbol{s}+\left(\frac{k_{R}+k_{V}}{k_{R}}\right)\left(-\frac{\Phi}{t_{g o}} \operatorname{sign}(\boldsymbol{s})+\boldsymbol{p}(t)\right)
\end{gathered}
$$

Now, substituting $\mathrm{k}_{\mathrm{R}}=6$ and $\mathrm{k}_{\mathrm{V}}=-2$ and assuming that $\Phi>\|\mathbf{p}\|$ we get:

$$
\frac{d}{d t} V=-\frac{4}{t_{g o}}\|\boldsymbol{s}\|^{2}-2 \boldsymbol{s}^{T}\left(\frac{\Phi}{t_{g o}} \operatorname{sign}(\boldsymbol{s})+\boldsymbol{p}(t)\right)=-\frac{2}{t_{g o}}\left(2\|\boldsymbol{s}\|^{2}+\Phi \boldsymbol{s}^{T} \operatorname{sign}(\boldsymbol{s})+\boldsymbol{s}^{T} \boldsymbol{p}(t)\right) \leq 0
$$

Therefore, Lyapunov function's derivative is shown to be negative definite. Moreover, the selected Lyapunov function (Eq.(35) is definite positive and radially unbounded. Finally, $V(\boldsymbol{s})$ is shown to be decrescent (see Appendix C). All the above conditions ensure global stability for the proposed OSG (See Appendix C for a formal statement of the theorem).

\section{Guidance Implementation and Simulations}

\section{A. OSG Implementation}

Figure 2 shows the schematic of a possible GNC architecture hosting the proposed guidance algorithm. The next generation of robotic spacecraft for autonomous close-proximity operations around asteroids will have the ability to autonomously perform navigation and guidance functions including landing site selection and obstacle detection and avoidance. Position and velocity determination comes from filters capable of processing optical navigation data (e.g. camera and LIDAR) to correctly estimate the relative position and velocity of the spacecraft around the asteroid. Attitude is determined using a combination of Inertial Measurement Unit (IMU) and Star Trackers. Position and velocity are fed to the guidance module which implements the OSG logic to determine the three components of the acceleration command with respect to the body-fixed, asteroid-centered reference frame. In the configuration shown 
in Fig. 2, it is assumed that the guidance and attitude functions are independent. More specifically, it is assumed that the only function of the attitude module is to maintain the body-fixed spacecraft reference frame aligned with the asteroid-centered frame. In this case, the guidance algorithm can generate three independent acceleration commands along the asteroid fixed directions.

The thrust vector is then quantized and limited to create the commanded thrust $\mathbf{T}_{\mathbf{C}}$ as follows:

$$
\boldsymbol{T}_{C}(i)=\left\{\begin{array}{ccc}
T_{\max } & \text { if } \boldsymbol{T}_{C}(i)>T_{\text {threshold }} \\
0 \text { if } T_{\text {threshold }}<\boldsymbol{T}_{C}(i)<T_{\text {threshold }} \\
-T_{\max } & \text { if } \boldsymbol{T}_{C}(i)<-T_{\text {threshold }}
\end{array}\right\} \text { for } i=1,2,3
$$

\section{B. OSG Performance Analysis}

The OSG algorithm is shown to theoretically guide the spacecraft to any desired state around the asteroid. The target state is defined as function of the close proximity operations required to accomplish a specific mission (e.g. landing, hovering). To fully test the ability of the algorithm to execute the assigned tasks, a realistic simulation environment describing the guided spacecraft dynamics around a selected asteroid of our choice is defined and implemented in MATLAB ${ }^{\circledR}$. The simulation environment describes the 3-D spacecraft motion in an asteroid bodyfixed reference frame (see Eq. (1)-(9)). Moreover, the following modeling assumptions have been considered:

1. The asteroid is assumed to wobble around its axis with a known nutation angle and spin rate.

2. The asteroid parameters (spin rate, nutation angle, density and dimensions) are assumed to be known.

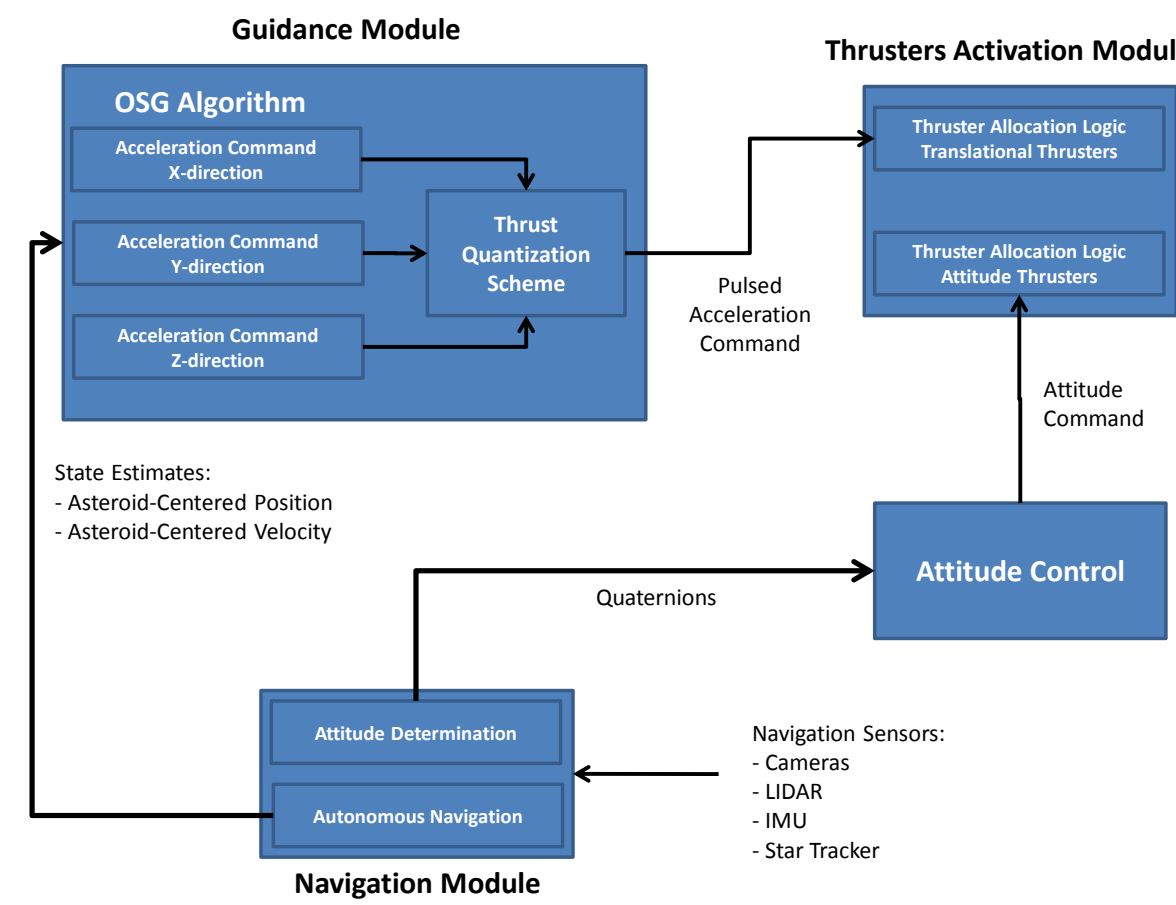

Figure 2.Block diagram representing the integration of the OSG algorithm into the spacecraft GNC subsystem for asteroid close-proximity operations

3. The spacecraft thrusters have a response to the guidance signal described by a first order dynamicswith a known time constant.

4. Mass flow rate errors are statistical in nature and modeled with a uniform distribution.

5. Sensor errors are assumed to be described by a Gaussian distribution with a known standard deviation. 
6. Gravity field is computed assuming the asteroid is described by a tri-axial ellipsoid of known dimensions. The known gravity field is perturbed by a Gaussian noise with a $10 \%$ standard deviation.

A set of Monte Carlo simulations have been executed to test the performance of the OGS algorithm in closeproximity operations scenarios typically planned during asteroid exploration missions. More specifically, we considered 1) a powered equatorial soft landing, where the spacecraft lands on a specified equatorial site of the selected asteroid and 2) a TAG maneuver, where the spacecraft touches the asteroid surface for a very short time. TAGs may be generally required to acquire an asteroid sample ${ }^{16}$. The guided maneuvers are assumed to be executed white operating around 1999 RQ36 "Bennu". Table 1 shows the nominal asteroid parameters describing the environment around Bennu. Table 2 shows the spacecraft parameters employed in the Monte Carlo simulations. Table 3 show the spacecraft initial conditions (the spacecraft is assumed to be in a terminator orbit) and final equatorial target state.

Table 1: 1999 RQ36 "Bennu" Nominal parameters

\begin{tabular}{|ccc|}
\hline Asteroid Parameter & Units & Values \\
\hline c-semi-axis & $\mathrm{m}$ & 250 \\
\hline b-semi-axis & $\mathrm{m}$ & 287 \\
\hline a-semi-axis & $\mathrm{m}$ & 350 \\
\hline angle between z-axis and angular velocity & 90 & degrees \\
\hline Density & $\mathrm{kg} / \mathrm{m}^{\wedge} 3$ & 1400 \\
\hline Magnitude of angular velocity & $\mathrm{Radians} / \mathrm{s}$ & $4.06 \mathrm{e}-4$ \\
\hline Mean Acceleration due to solar radiation & $\mathrm{m} / \mathrm{s}^{\wedge} 2$ & $1.11963 \mathrm{e}-7$ \\
\hline $\begin{array}{c}\text { Standard Deviation Acceleration due to solar } \\
\text { radiation }\end{array}$ & $\mathrm{m} / \mathrm{s}^{\wedge} 2$ & $3.00000 \mathrm{e}-8$ \\
\hline
\end{tabular}

Table 2: Spacecraft Nominal Parameters

\begin{tabular}{|ccc|}
\hline Spacecraft Parameter & Units & Values \\
Mass & $\mathrm{Kg}$ & 750 \\
\hline Engine mass flow rate variation & $\mathrm{Kg} / \mathrm{sec}$ & $10 \%$ \\
\hline Engine actuator delay & $\mathrm{Sec}$ & 0.25 \\
\hline Estimation errors (position and velocity) & meters, $\mathrm{m} / \mathrm{sec}$ & $5 \%$ \\
\hline
\end{tabular}

Table 3: Initial and Target States

\begin{tabular}{|ccc|}
\hline Trajectory Parameter & Units & Values \\
\hline Position where spacecraft leaves orbit & $\mathrm{m}$ & $(1500,0,0)+\mathrm{U}(-100,100)$ \\
\hline Velocity where spacecraft leaves orbit & $\mathrm{m} / \mathrm{s}$ & $(-0.04,-0.047,-0.079)+\mathrm{U}(-0.02,0.02)$ \\
\hline Landing Position & $\mathrm{m}$ & $(0,-287,0)$ \\
\hline Landing Velocity & $\mathrm{m} / \mathrm{s}$ & $(0,0,0)$ \\
\hline
\end{tabular}

\section{OSG AsteroidSoft Landing}

In the first set of Monte Carlo tests for the OSG algorithm, we considered a scenario where the spacecraft executes a guided autonomous landing in the equatorial region of Bennu. The spacecraft is assumed to leave a nominal terminator orbit and autonomously employs OSG to navigate toward the desired state. A waypoint navigation approach is employed. More specifically, the spacecraft targets an initial state (waypoint) located at an intermediate position between the initial state and final target state. The OSG targets the waypoint first and, once achieved it within a specified tolerance switches to the final target as desired target. Table 4 shows the initial, intermediate waypoint and final state.

A set of 1000 Monte Carlo simulations has been executed to analyze the guidance performances. Importantly, the spacecraft's mass is varied randomly between its nominal value and $10 \%$ less than this nominal value (uniform 
distribution). The asteroid's nutation angle, density, and angular velocity are also varied $+/-10 \%$ from their nominal values (uniform distribution). The latter reflects possible modeling errors in the measurement of the asteroid's dynamics. To further stress the proposed OSG algorithm we increased the mean and standard deviation of the acceleration due to solar radiation pressure to $0.0001 \mathrm{~m} / \mathrm{s}^{2}$ and $0.00001 \mathrm{~m} / \mathrm{s}^{2}$ respectively. A statistical model that accounts for navigation error have been considered (5\% standard deviation, 1-sigma, see Table 2). The guidance algorithm has been implemented and pulsed with $10 \mathrm{~Hz}$ frequency.

Table 4: Initial and Target States

\begin{tabular}{|c|c|c|}
\hline \multicolumn{3}{|c|}{ Leave Orbit } \\
\hline Position (m) & Velocity $(\mathrm{m} / \mathrm{s})$ & Time of flight $(\mathrm{s})$ \\
\hline$(1500,0,0)+U(-100,100)$ & $(-0.04,-0.047,-0.079)+U(-0.02,0.02)$ & 4000 \\
\hline \multicolumn{3}{|c|}{ First Waypoint } \\
\hline Position (m) & Velocity (m/s) & Time of flight $(\mathrm{s})$ \\
\hline$(150,-350,0)$ & $(0.05,0.05,0.0)$ & 1000 \\
\hline \multicolumn{3}{|c|}{ Landing } \\
\hline Position (m) & Velocity $(\mathrm{m} / \mathrm{s})$ & Time of flight $(\mathrm{s})$ \\
\hline$(0,-287,0)$ & $(0,0,0)$ & $\mathrm{N} / \mathrm{A}$ \\
\hline
\end{tabular}
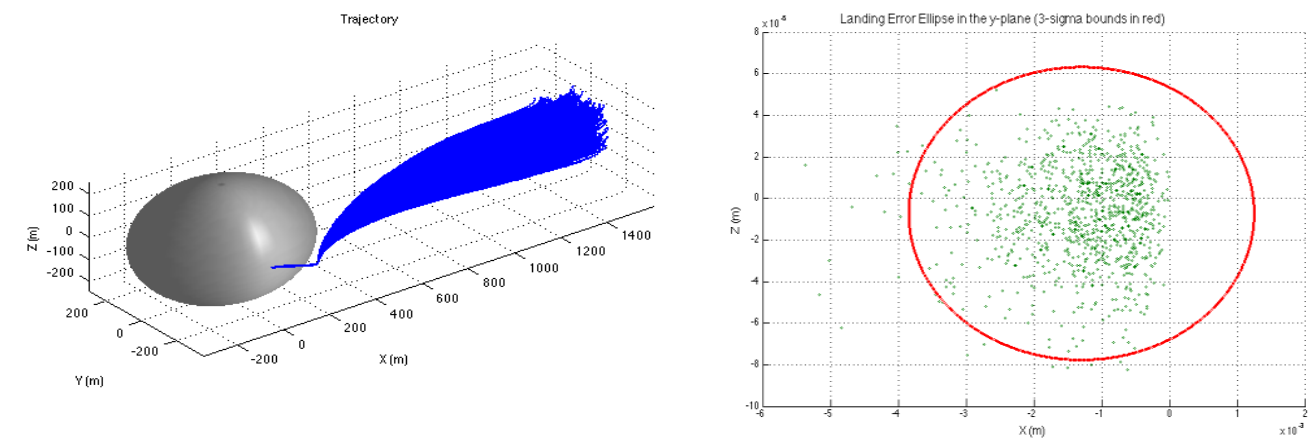

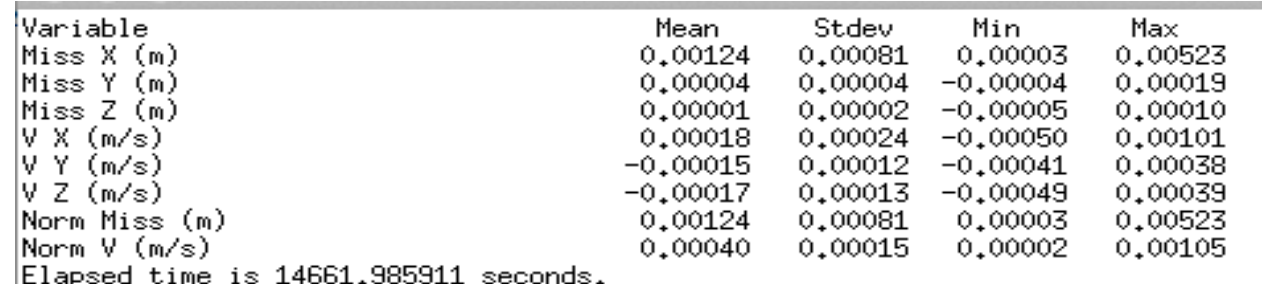

Figure 3.Monte Carlo simulations results. Top Left: 3-D guided trajectories. Top Right: Landing spots and landing error ellipse (1-sigma). Bottom: Monte Carlo statistics

Figure 3 shows the results of the OSG-guided Monte Carlo simulations, reporting 3-D trajectories, the landing error ellipse (1-sigma) and the statistics associated with the landing errors. OSG is shown to perform very well in spite of the uncertain environment. Position and velocity errors are shown to be extremely low, which is an indication of the ability of the guidance algorithm to drive the spacecraft to the desired target with pinpoint accuracy. 


\section{OSG for TAG Maneuvers}

The second set of Monte Carlo simulations has been implemented to simulate an autonomous TAG maneuver similar to the one designed for the upcoming NASA OSIRIS REx asteroid sample return mission. Touch-And-Go (TAG) maneuvers have been specifically conceived to touch the asteroid surface for a very short time required to collect a sample via the acquisition mechanism. Importantly, the trajectory includes a final unpowered terminal descent necessary to minimize sample contamination. The OSIRIS REx Flight Dynamics Team designed a set of three open-loop maneuvers aiming at satisfying the currently projected landing error requirement $(<25$ meters $)$. The sequence includes a) an initial burn required to leave the terminator (parking) orbit, b) an intermediate burn (checkpoint) at $125 \mathrm{~m}$ altitude that target a point approximately 30 meters above the desired site with a vertical (descent) velocity less than $10 \mathrm{~cm} / \mathrm{sec}$ and c) a final burn (matchpoint) that matches the asteroid angular velocity and initiates the vertical descent toward the asteroid surface. Importantly, thrusters are activated after the sample acquisition to escape the asteroid surface. The TAG maneuver sequence is designed on the ground and command uploaded after careful testing in a comprehensive simulation environment. Nevertheless, a limited level of autonomy is implemented on-board ${ }^{16}$.Indeed, the on-board GNC LIDAR is activated for asteroid limb detection which provides range-to-go information and altitude measurements. Range-to-go and one single altitude sample are fed to an algorithm that adjusts burn magnitude, direction and timing to reduce further the TAG error ellipse ${ }^{16}$.

Table 5: TAG waypoints

\begin{tabular}{|c|c|c|}
\hline \multicolumn{3}{|c|}{ Leave Orbit } \\
\hline Position $(\mathrm{m})$ & Velocity $(\mathrm{m} / \mathrm{s})$ & Time of flight (s) \\
\hline$(1500,0,0)+U(-100,100)$ & $\begin{array}{c}(-0.04,-0.047,-0.079)+U(-0.02 \\
0.02)\end{array}$ & 4000 \\
\hline \multicolumn{3}{|c|}{ First Waypoint } \\
\hline Position (m) & Velocity $(\mathrm{m} / \mathrm{s})$ & Time of flight (s) \\
\hline$(150,-350,0)$ & $(0.0,0.0,0.0)$ & 800 \\
\hline \multicolumn{3}{|c|}{ Second Waypoint } \\
\hline Position (m) & Velocity $(\mathrm{m} / \mathrm{s})$ & Time of flight (s) \\
\hline$(12,-317,8)$ & $(0.0,0.0,0.0)$ & Unconstrained \\
\hline \multicolumn{3}{|c|}{ Landing } \\
\hline Position (m) & Velocity $(\mathrm{m} / \mathrm{s})$ & Time of flight (s) \\
\hline$(0,-287,0)$ & $(0,0,0)$ & N/A \\
\hline
\end{tabular}

A similar a TAG maneuver scenario around Bennu has been implemented in our simulation environment to test the proposed guidance algorithm. In this case OSG is designed to target a set of two waypoints (checkpoint and matchpoint) defined to implement a TAG sequence for an equatorial landing. Table 5 shows the initial, intermediate and final target states. Importantly, the two intermediate waypoints have been designed such that the waypoint prior to the landing is located $30 \mathrm{~m}$ above the intended sampling site. Once the $30 \mathrm{~m}$ altitude is achieved, the spacecraft is allowed to fall toward the surface in an un-powered fashion. Note that the waypoint is selected to be not exactly above the desired landing point but somewhat offset to allow for drift caused by the asteroid's rotation and the solar radiation pressure (estimated via modeling). 
Table 6: TAG sampling statistics

\begin{tabular}{lrrrr}
\hline Variable & Mean & Stdev & \multicolumn{1}{c}{ Min } & \multicolumn{1}{c}{ Max } \\
Miss $X(\mathrm{~m})$ & 0.23596 & 0.48409 & -1.47761 & 1.89947 \\
Miss $Y(\mathrm{~m})$ & -0.00313 & 0.00174 & -0.00616 & -0.00001 \\
Miss $Z(\mathrm{~m})$ & 0.36156 & 0.18830 & -0.25051 & 0.91376 \\
$V X(\mathrm{~m} / \mathrm{s})$ & 0.01158 & 0.00038 & 0.01020 & 0.01293 \\
$V Y(\mathrm{~m} / \mathrm{s})$ & -0.06174 & 0.00007 & -0.06195 & -0.06151 \\
$V Z(\mathrm{~m} / \mathrm{s})$ & 0.02467 & 0.00016 & 0.02414 & 0.02515 \\
Norm Miss $(\mathrm{m})$ & 0.61481 & 0.27935 & 0.06548 & 1.97137 \\
Norm $\mathrm{V}(\mathrm{m} / \mathrm{s})$ & 0.06749 & 0.00014 & 0.06701 & 0.06788
\end{tabular}

A set of 1000 Monte Carlo simulations has been executed to analyze the guidance performances. As in the precious case, the spacecraft's mass is varied randomly between its nominal value and $10 \%$ less than this nominal value (uniform distribution). The asteroid's nutation angle, density, and angular velocity are also varied $+/-10 \%$ from their nominal values (uniform distribution). The latter reflects possible modeling errors in the measurement of the asteroid's dynamics. To further stress the proposed OSG algorithm we increased the mean and standard deviation of the acceleration due to solar radiation pressure to $0.0001 \mathrm{~m} / \mathrm{s}^{2}$ and $0.00001 \mathrm{~m} / \mathrm{s}^{2}$ respectively. A statistical model that accounts for navigation error have been considered (5\% standard deviation, 1-sigma, see Table 2). The guidance algorithm has been implemented and pulsed with $1 \mathrm{~Hz}$ frequency. Figure 4 shows the 3-D Monte Carlo guided trajectories, the sampling error ellipse (1-sigma) and the histograms for the position and velocity errors. Table 6 reports details of the sampling error statistics. Figure 5 reports selected telemetry data for the case where the engine actuator delay time constant is 0 s. This is equivalent to viewing the commanded thrust as opposite to the real thrust delayed by the time-response of the thruster.

The results indicate that the proposed OSG algorithm is capable of implementing pinpoint accuracy $(<1 \mathrm{~m}, 1$ sigma) way beyond conventional open-loop TAG requirements.
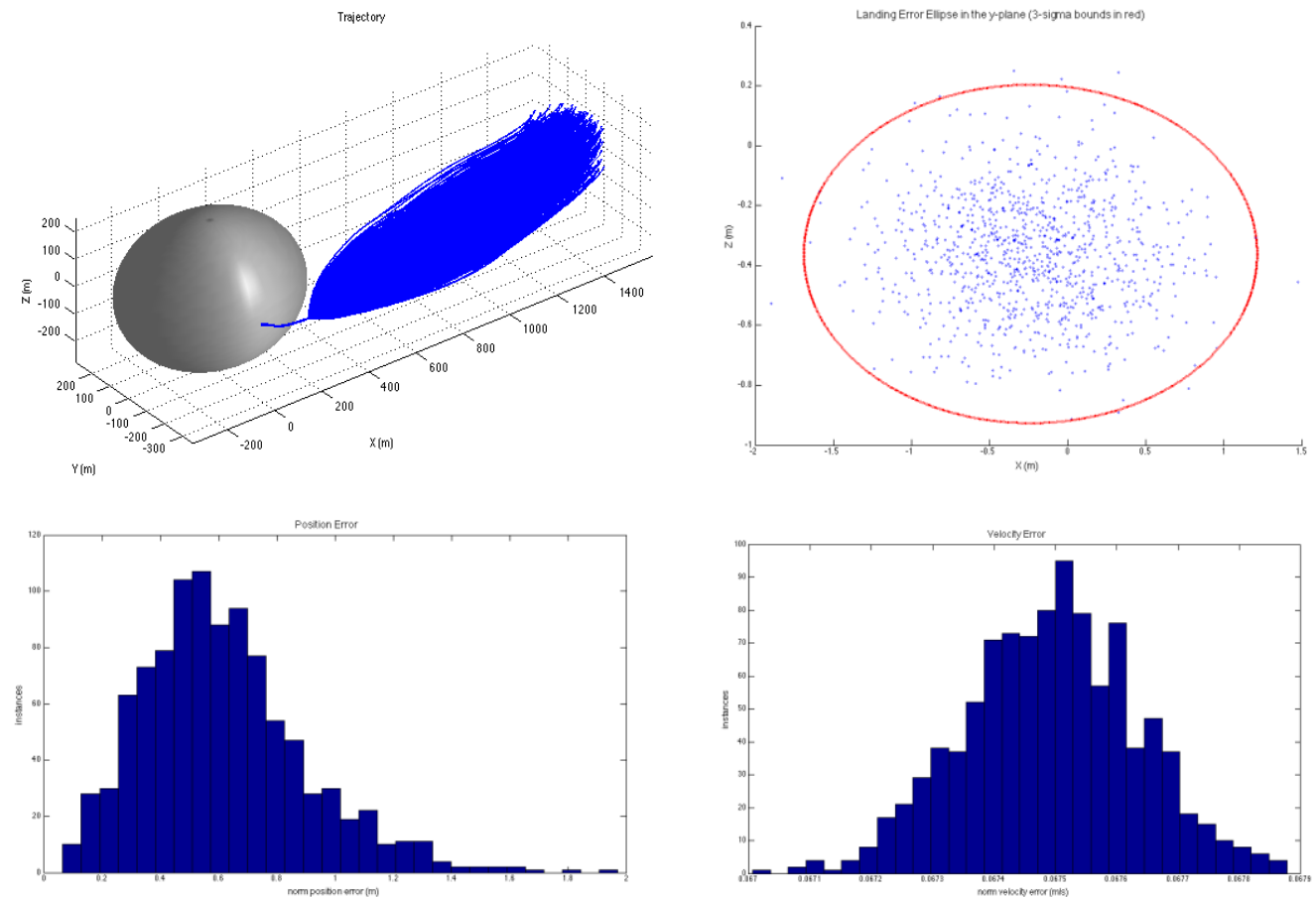

Figure 4. Monte Carlo simulations for the TAG maneuver. Top Left: 3-D guided trajectories. Top Right: TAG surface sampling spots and TAG error ellipse (1-sigma). Bottom Left: Histogram of the position error norm. Bottom Right: Histogram of the velocity error norm 


\section{Conclusions and Future Efforts}

In this paper, the theoretical development of a class of non-linear guidance algorithm for autonomous asteroid close-proximity operations has been discussed. The guidance design approach is based on a combination of optimal control theory and sliding control theory, yielding what has been named Optimal Sliding Guidance (OSG). Indeed, the generalized ZEM/ZEV feedback guidance is augmented by a sliding mode to ensure global stability. Importantly, the guidance law is naturally derived from the definition of an appropriate sliding surface that includes a combination of ZEM and ZEV. OSG has been tested in a set of realistic scenarios representing situations typically encounters in close-proximity operations around small bodies in general and asteroids in particular. The guidance algorithm is shown to perform well in a pulsed mode achieving pinpoint accuracy. Importantly, such class of algorithms may be functional to future mission where stringent sampling or landing requirements are required. Integration and testing of OSG with navigation algorithms (e.g. optical and LIDAR-based navigation) is currently underway.
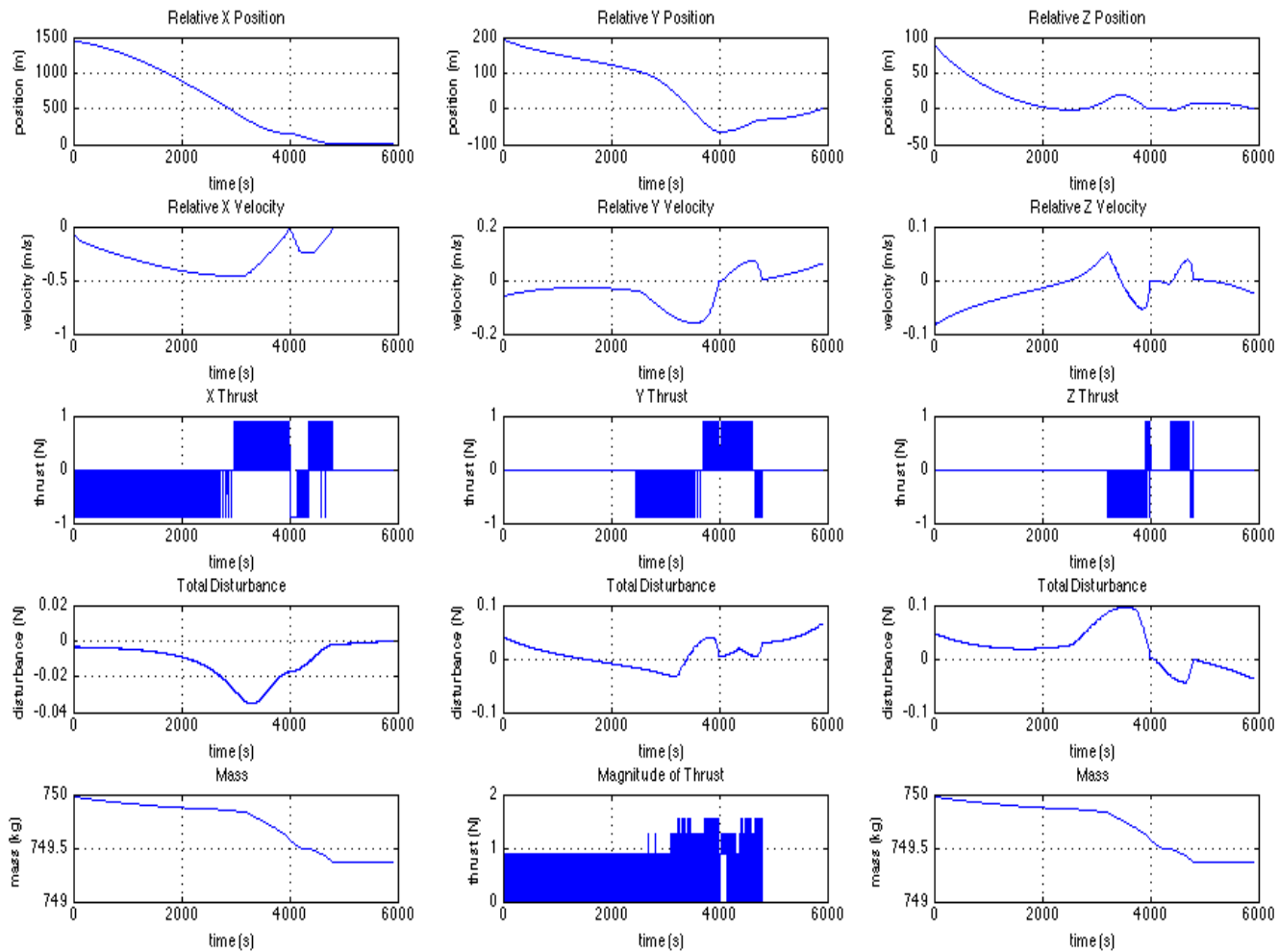

Figure 5.Selected telemetry data for the case where the engine actuator delay time constant is 0s (acceleration command)

\section{Appendix}

\section{A. Derivation of the ZEM/ZEV guidance law from Calculus of Variations}

In this section, we apply optimal control theory to derive the optimal guidance equations (OGL, Eq. (21)). The optimal control problem can be formulated as follows:

Find the spacecraft acceleration command $\boldsymbol{a}_{\text {Сомм }}(t)$ that minimizes the performance index $J$ 


$$
J=\frac{1}{2} \int_{t}^{t_{f}} a_{\text {СомM }}^{T}(\tau) a_{\text {СомM }}(\tau)
$$

Subject to

$$
\left\{\begin{array}{c}
\dot{\boldsymbol{r}}_{L}=\boldsymbol{v}_{L} \\
\dot{v}_{L}=a_{L}=g\left(r_{L}, t\right)+a_{\text {COMM }}(t)
\end{array}\right.
$$

With boundary conditions described as

$$
\left\{\begin{aligned}
r_{L}(t) & =r_{L} \\
r_{L}\left(t_{f}\right) & =r_{L_{f}} \\
v_{L}(t) & =v_{L} \\
v_{L}\left(t_{f}\right) & =v_{L_{f}}
\end{aligned}\right.
$$

Here, we apply calculus of variations to determine the necessary conditions for an extremal. Define the admissible functions as follows:

$$
\left\{\begin{array}{c}
\boldsymbol{r}_{L}(t, \alpha)=\boldsymbol{r}_{L}(t)+\alpha \boldsymbol{h}(t) \\
\boldsymbol{v}_{L}(t, \alpha)=\boldsymbol{v}_{L}(t)+\alpha \dot{\boldsymbol{h}}(t) \\
\boldsymbol{a}_{L}(t, \alpha)=\boldsymbol{a}_{\text {COMM }}(t)+\alpha \ddot{\boldsymbol{h}}(t)
\end{array}\right.
$$

Where

$$
\left\{\begin{array}{l}
\boldsymbol{h}(t)=\boldsymbol{h}\left(t_{f}\right)=\mathbf{0} \\
\dot{\boldsymbol{h}}(t)=\dot{\boldsymbol{h}}\left(t_{f}\right)=\mathbf{0} \\
\ddot{\boldsymbol{h}}(t)=\ddot{\boldsymbol{h}}\left(t_{f}\right)=\mathbf{0}
\end{array}\right.
$$

The cost function is now a function of $\alpha$

$$
J(\alpha)=\frac{1}{2} \int_{t}^{t_{f}} \boldsymbol{a}_{\text {СОМM }}^{T}(\tau) \boldsymbol{a}_{\text {СОмM }}(\tau) d \tau+\alpha \int_{t}^{t_{f}} \boldsymbol{a}_{\text {СОM }}^{T}(\tau) \ddot{\boldsymbol{h}}(\tau) d \tau+\frac{\alpha^{2}}{2} \int_{t}^{t_{f}} \ddot{\boldsymbol{h}}^{T}(\tau) \ddot{\boldsymbol{h}}(\tau) d \tau
$$

The necessary condition for $J(\alpha)$ to be a minimum is written as

$$
\left.\frac{d J}{d \alpha}\right|_{\alpha=0}=\int_{t}^{t_{f}} \boldsymbol{a}_{\text {COMM }}^{T}(\tau) \ddot{\boldsymbol{h}}(\tau) d \tau=0
$$

Integrating by parts twice we obtain:

$$
\int_{t}^{t_{f}} \boldsymbol{a}_{\text {COMM }}^{T} \boldsymbol{a}_{\text {СOM }} d \tau=-\left.\frac{d}{d t} \boldsymbol{a}_{\text {COMM }}^{T}(\tau) \dot{\boldsymbol{h}}(\tau)\right|_{t} ^{t_{f}}+\int_{t}^{t_{f}} \frac{d^{2}}{d \tau^{2}} \boldsymbol{a}_{\text {COMM }}^{T}(\tau) \boldsymbol{h}(\tau) d \tau=\int_{t}^{t_{f}} \frac{d^{2}}{d \tau^{2}} \boldsymbol{a}_{\text {COMM }}^{T}(\tau) \boldsymbol{h}(\tau) d \tau
$$

Consequently:

$$
\frac{d J}{d \alpha}=0 \quad=>\int_{t}^{t_{f}} \frac{d^{2}}{d \tau^{2}} \boldsymbol{a}_{\text {COMM }}^{T}(\tau) \boldsymbol{h}(\tau) d \tau=0
$$

We now use the fundamental lemma of calculus of variations

$$
\frac{d^{2}}{d t^{2}} \boldsymbol{a}_{\text {COMM }}^{T}=\mathbf{0}^{T} \quad=>\boldsymbol{a}_{\text {СOMM }}(t)=\boldsymbol{A}_{1} t-\boldsymbol{A}_{2}
$$

To express the acceleration command as a function of ZEM and $\mathbf{Z E V}$, we need to determined $\boldsymbol{A}_{1}$ and $\boldsymbol{A}_{2}$. Eq. (A10) is replaced in Eq. (A3) and the equations of motion are integrated between $t$ and $t_{f}$ : 


$$
\begin{gathered}
\boldsymbol{v}_{L}(t)=\boldsymbol{v}_{L_{f}}-\frac{A_{1}}{2}\left(t_{f}+t\right) t_{g o}+\boldsymbol{A}_{2} t_{g o}+\int_{t}^{t_{f}} \boldsymbol{g}\left(\boldsymbol{r}_{L}, \tau\right) d \tau \\
\boldsymbol{r}_{L}(t)=\boldsymbol{r}_{L_{f}}-\boldsymbol{v}_{L}(t) t_{g o}-\frac{A_{1}}{6}\left(2 t+t_{f}\right) t_{g o}^{2}+\frac{A_{2}}{2} t_{g o}^{2}-\int_{\mathrm{t}}^{t_{f}} \int_{\tau^{\prime}}^{t_{f}} \boldsymbol{g}\left(\boldsymbol{r}_{L}, \tau^{\prime}\right) d \tau^{\prime} d \tau
\end{gathered}
$$

Eq. (A11) and Eq. (A12) can be inverted to determine $\boldsymbol{A}_{1}$ and $\boldsymbol{A}_{2}$. Remembering the definition of ZEM and ZEV (Eq. (17-18)) we have:

$$
\begin{gathered}
\boldsymbol{A}_{1}=-\frac{12}{t_{g o}^{3}}\left(\boldsymbol{Z E} \boldsymbol{M}-\frac{1}{2} t_{g o} \boldsymbol{Z} \boldsymbol{E} \boldsymbol{V}\right) \\
\boldsymbol{A}_{2}=-\frac{6}{t_{g o}^{3}}\left[\left(t+t_{f}\right) \boldsymbol{Z E} \boldsymbol{M}-\frac{1}{3} t_{g o}\left(2 t+t_{f}\right) \boldsymbol{Z} \boldsymbol{E} \boldsymbol{V}\right]
\end{gathered}
$$

The OGL, i.e. the optimal acceleration command is determined by replacing Eq. (A13) and Eq. (A14) into Eq. (A10):

$$
\begin{gathered}
\boldsymbol{a}_{\text {СомM }}(t)=-\frac{12}{t_{g o}^{3}}\left(\boldsymbol{Z E M}-\frac{1}{2} t_{g o} \boldsymbol{Z E V}\right) t+\frac{6}{t_{g o}^{3}}\left[\left(t+t_{f}\right) \boldsymbol{Z E M}-\frac{1}{3} t_{g o}\left(2 t+t_{f}\right) \boldsymbol{Z E V}\right]= \\
=-\frac{2}{t_{g o}} \boldsymbol{Z E V}+\frac{6}{t_{g o}^{2}} \boldsymbol{Z E M}
\end{gathered}
$$

Finally, setting $k_{v}=-2$ and $k_{R}=6$, we get:

$$
\boldsymbol{a}_{\text {СомM }}(t)=\frac{k_{R}}{t_{g o}^{2}} \boldsymbol{Z E M}(t)+\frac{k_{v}}{t_{g o}} \boldsymbol{Z E V}(t)
$$

\section{B. Sliding Surface Non-linear Dynamics}

Consider a sliding surface with the following non-linear, first-order dynamics (see also Eq.(29)):

$$
\frac{d}{d t} \boldsymbol{s}=-K(t) \boldsymbol{s}=-\frac{4}{t_{g o}} \boldsymbol{s}=-\frac{4}{t_{g o}} \boldsymbol{S}
$$

By using Eq.(29), Eq.(30) and Eq.(31) and remembering that $\mathrm{kR}=6$ and $\mathrm{kV}=-2$, Eq.(B1) becomes an explicit function of the time-to-go or $t_{F}-t$ :

$$
\frac{d}{d t} \boldsymbol{S}=-\frac{4}{t_{g o}} \boldsymbol{s}=-\frac{4}{t_{F}-t} \boldsymbol{S}
$$

Here, we show that the system reaches the sliding surface in a finite time and exactly when $t=t_{F}$. Assume that the initial conditions are stated as $(0)=\boldsymbol{s}_{\mathbf{0}}$. By applying the separation of variables we obtain:

$$
\frac{d s_{i}}{s_{i}}=-\frac{4 d t}{t_{F}-t}
$$

Where $\mathrm{i}=1,2,3$ are the components of the sliding surface vector. Eq.(B3) can be integrated to obtain:

$$
\log \left(s_{i}\right)=4 \log \left(t_{F}-t\right)+C_{i}
$$

By imposing the initial conditions and taking the exponential of both sides, the solution becomes:

$$
\begin{gathered}
s_{i}(t)=s_{i 0}\left(t_{F}-t\right)^{4} \\
\boldsymbol{s}(t)=\boldsymbol{s}_{\mathbf{0}}\left(t_{F}-t\right)^{4}
\end{gathered}
$$

The derivative of the sliding surface vector can be computed explicitly: 


$$
\dot{\boldsymbol{s}}(t)=4 \boldsymbol{s}_{0}\left(t_{F}-t\right)^{3}
$$

Eq.(B5)-(B7) are analytical expressions for the sliding surface vector and its derivative. Since the exponent of the RHS of both Eq.(B6) and Eq.(B7) is greater than zero, the surface will approach zero as $t \rightarrow t_{F}$. More specifically, the surface is reached exactly when $t=t_{F}$.

\section{Global Stability Analysis of the OSG}

ReferencesTo analyze the global stability of the proposed OSG algorithm, we rely on the following Lyapunov's stability theorem [21]:

Uniform Global Asymptotic Stability for Non-Autonomous Systems: If in the whole state space, there exists a function $V(s, t)$ with continuous partial derivatives such that the following conditions are satisfied:
1. $V(\boldsymbol{s}, t)$ is positive definite
2. $\dot{V}(s, t)$ is negative definite
3. $V(\boldsymbol{s}, t)$ is decrescent
4. $V(s, t)$ is radially bounded

Then the origin is uniformly globally asymptotically stable.(For the proof, see Slotine and Li [21] page 107).

In our case, the system reaches the origin in a finite time $t_{F}$ and no switching (and consequently no chattering) is possible during the motion, i.e. the sliding surface is reached for the first time at the landing point (end of the flight). One of the key point is to show that the selected Lyapunov function (Eq.(34)) is decrescent. Using the analytical result obtained in Appendix $\mathrm{B}$, it is possible to obtain an analytical expression for both the selected Lyapunov function and its derivative. Inserting Eq.(B6)-(B7) into Eq.(34) and Eq.(37) and ignoring the perturbations, we obtain:

$$
\begin{gathered}
V=\frac{1}{2}\left\|\boldsymbol{s}_{\mathbf{0}}\right\|^{2}\left(t_{F}-t\right)^{8} \\
\dot{V}=-\frac{4}{t_{F}-t}\left\|s_{0}\right\|^{2}\left(t_{F}-t\right)^{8}-\frac{2}{t_{F}-t} \Phi\left(\left|s_{10}\right|+\left|s_{20}\right|+\left|s_{30}\right|\right)\left(t_{F}-t\right)^{4}= \\
=-4\left\|s_{0}\right\|^{2}\left(t_{F}-t\right)^{7}-2 \Phi\left(\left|s_{10}\right|+\left|s_{20}\right|+\left|s_{30}\right|\right)\left(t_{F}-t\right)^{3}
\end{gathered}
$$

The Lyapunov function is obviously decrescent with time and goes to zero as $t \rightarrow t_{F}$. However, the statement can be formally proven by finding a uniform function that bounds $\dot{V}$ :

$$
\dot{V}=-4\left\|s_{0}\right\|^{2}\left(t_{F}-t\right)^{7}-2 \Phi\left(\left|s_{10}\right|+\left|s_{20}\right|+\left|s_{30}\right|\right)\left(t_{F}-t\right)^{3} \leq-4\left\|s_{0}\right\|^{2}\left(t_{F}-t\right)^{7}
$$

The uniform global asymptotic stability follows with $W(s)=4\left\|s_{0}\right\|^{2}\left(t_{F}-t\right)^{7}$

\section{References}

${ }^{1}$ F. J. Ciesla, S. B. Charnley, The physics and chemistry of nebular evolution, in: Lauretta, D.S., McSween, H.Y. (Eds.), Meteorites and the Early Solar System II. University of Arizona Press, Tucson, pp.209-230, 2006.

${ }^{2}$ J. S. Kargel, Metalliferous asteroids as potential sources of precious metals, J. Geophys. Res. 99, (1994) pp. 21-129

${ }^{3}$ National Research Council, Defending Planet Earth: Near-Earth Object Surveys and Hazard Mitigation Strategies, Final Report, Committee to Review Near-Earth Object Surveys and Hazard Mitigation Strategies, The National Academies Press, January 2010. 
${ }^{4}$ Lauretta, Dante et al., “An Overview of the OSIRIS-REx Asteroid Sample Return Mission” [\#2491], 42th Lunar and Planetary Science Conference Abstracts, Lunar and Planetary Institute, Houston, Texas, 2012

${ }^{5}$ Lara, M., and Scheeres, D. J., "Stability Bounds for Three-Dimensional Motion Close to Asteroids," Journal of the Astronautical Sciences, Vol. 50, No. 4, (2002), pp. 389-409.

${ }^{6}$ Miso, T., Tatsuaki Hashimoto, and KeikenNinomiya."Optical guidance for autonomous landing of spacecraft." Aerospace and Electronic Systems, IEEE Transactions on 35.2 (1999): 459-473.

${ }^{7}$ Shuang, Li, and Cui Pingyuan. "Landmark tracking based autonomous navigation schemes for landing spacecraft on asteroids." ActaAstronautica 62.6 (2008): 391-403.

${ }^{8}$ Bhaskaran, Shyam, et al. "Small Body Landings Using Autonomous Onboard Optical Navigation." The Journal of the Astronautical Sciences 58.3 (2011): 409-427.

${ }^{9}$ Furfaro, Roberto, Dario Cersosimo, and Daniel R. Wibben."Asteroid Precision Landing via Multiple Sliding Surfaces Guidance Techniques." Journal of Guidance, Control, and Dynamics (2013): 1-18.

${ }^{10}$ Levant, Arie. "Principles of 2-sliding mode design." Automatica 43.4 (2007): 576-586.

${ }^{11}$ Guo, Yanning, Matt Hawkins, and Bong Wie."Applications of Generalized Zero-Effort-Miss/Zero-Effort-Velocity Feedback Guidance Algorithm." Journal of Guidance, Control, and Dynamics 36.3 (2013): 810-820.

${ }^{12}$ Battin, Richard H.An introduction to the mathematics and methods of astrodynamics.Aiaa, 1999.

${ }^{13}$ D’Souza, Christopher N. "An optimal guidance law for planetary landing." Paper No. AIAA (1997): 97-3709.

${ }^{14}$ Hawkins, Matt, YanningGuo, and Bong Wie. "ZEM/ZEV Feedback Guidance Application to Fuel-Efficient Orbital Maneuvers Around an Irregular-Shaped Asteroid." AIAA Guidance, Control, and Navigation Conference, Minneapolis, Minnesota. 2012.

${ }^{15}$ Slotine, Jean-Jacques E., and Weiping Li. Applied nonlinear control.Vol. 199.No. 1. New Jersey: Prentice hall, 1991.

${ }^{16}$ Berry, Kevin, et al. "OSIRIS-REx Touch-And-Go (TAG) Mission Design and Analysis." (2013), AAS 13-095 